\title{
Vehicle Routing Problem Based on Improved Particle Swarm Optimization
}

\author{
Chunyan Qiu ${ }^{1,2, a}$, Xi Yang' and Yang Liu ${ }^{1, b, *}$ \\ ${ }^{1 .}$ School of Management Science and Information Engineering, Jilin University of Finance and Economics, \\ Changchun 130117, China \\ ${ }^{2 .}$ Key Laboratory of Logistics Industry Economy and Intelligent Logistics, Jilin University of Finance and \\ Economics, Changchun 130117 China \\ a email:81692002@qq.com, ${ }^{\mathrm{b}}$ email: 7923759@qq.com \\ *Corresponding author
}

Keywords: Vehicle Routing, Optimization, Particle Swarm Algorithm

\begin{abstract}
The use of intelligent optimization algorithms to optimize vehicle routing problem has become a hot topic in international researches. The normal particle swarm optimization (PSO) algorithm is a validated evolutionary computation way of searching the extreme of function, which is simple in application and quick in convergence, but low in precision and easy in premature convergence. In this paper, the improved particle swarm optimization algorithm is used to optimize the logistics vehicle path by setting the inertia factor to 0 . Through the simulation experiment analysis, the improved particle swarm optimization algorithm has better convergence (linear convergence). This algorithm avoids the problem that the global optimal is replaced by the local value, reduces the number of iterations required for search in optimal solution, and shortens the optimization time.
\end{abstract}

\section{Introduction}

The Vehicle Routing Problem (VRP) is derived from transportation which is the core problem of transportation organization optimization that first proposed by Dantzing and Ramser in 1959[1], and it is also a classic combination optimization problem for the operations research.The intelligent optimization algorithm is a meta-heuristic algorithm based on the principle of physics or bionics, such as the simulated annealing algorithm, the tabu search algorithm, the genetic algorithm, the particle swarm optimization algorithm, etc. The intelligent optimization algorithm can solve the optimal path of the vehicle in a limited time.Since particle swarmoptimization performs well insearching optimal solutionof vehicle routing problems, it has become a common algorithm for solving vehicle routing problems[2].PSO has a good performance in solving PVRand has gradually gained widespread attention in the academic community.

\section{Basic problem description and model establishment}

\subsection{Description of the problem}

Vehicle path optimization is also a traveling salesman's problem which means that the path taken by each vehicle is optimized in order to achieve the shortest overall path.

\subsection{Model establishment}

This paper references the model [3] and briefly describes the problem as:there are $l$ warehouses (represented by $1,2 \ldots . . l)$ and $k$ vehicles ( $k$ represents the $k$ th car) whose capacity is $q_{k}(k=1,2, \ldots . . . K)$. The model is built as follows:

$X_{i k}=\{0,1\}$ indicates that the value is 1 when the warehouses from $L_{i}$ to $L_{j}$ are served by the $k$ th car, otherwise the value is 0 ;

$Y_{i k}=\{0,1\}$ indicates that the value is 1 when the $L_{i}$ warehouse is served by the $k$ th car, otherwise 
the value is 0 ;

$$
\begin{aligned}
& \text { Length }=\sum_{i=0}^{n} \sum_{j=0}^{n} \sum_{k=1}^{k} c_{i j} x_{i j k} . \\
& \sum_{\mathrm{i}=1}^{n} d_{i} y_{i k} \leq \mathrm{q}, k=0,1,2 \ldots . . K \\
& \sum_{i=0}^{n} \sum_{k=1}^{k} x_{\mathrm{ijk}}=1, j=0,1 \ldots . . n \\
& \sum_{\mathrm{j}=0}^{n} \sum_{k=1}^{k} x_{i j k}=1, i=0,1 \ldots . . n
\end{aligned}
$$

Where:

$d_{i}$ is the freight volume of the $L$ th warehouse;

$L_{i}$ is the ith warehouse;

$c_{i j}$ is the distance from the point $L_{i}$ to the point $L_{j}$;

$q$ is the capacity of the vehicle.

\section{Particle Swarm intelligence algorithm for solving the logistics vehicle routing}

\subsection{The Mathematical Description and Limitations of the Particle Swarm Optimization Algorithm}

The particle swarm optimization algorithm is an intelligent algorithm proposed by simulating the predation behavior of birds[4].The working principles of the PSO algorithm are as follows:

Firstly, initialize a set of random solutions and complete self-renewalby iteratively tracking two extremum values.The two extremum values include the local extremum points (represented by the $p \_i d$ ) and the global extremum points (represented by the $p \_g d$ );

Then during each iteration, the particles update the speed and position by the following formulas.

$$
\begin{aligned}
& V_{\text {id }}(t+1)=w^{*} V_{\text {id }}(t)+c_{1} * \text { rand }()\left(p_{\text {id }}(t)-X_{\text {id }}(t)\right)+c_{2}()\left(p_{g d}(t)-X_{\text {id }}(t)\right) . \\
& X_{\text {id }}(\mathrm{t}+1)=X_{\text {id }}(t)+V_{\text {id }}(t+1) .
\end{aligned}
$$

Where:

$X=\left(X_{i 1}, X_{i 2} \ldots \ldots X_{i d}\right)$ is a particles set;

$V_{i}=\left(V_{i 1}, V_{i 2} \ldots \ldots V_{i d}\right)$ indicates the flying speed of the particle in space (The flying speed of the $i$ particle in the $d$ th dimension);

$w$ represents the inertia factor;

$c_{1}$ and $c_{2}$ are the acceleration factors which are also known as the learning factors.

The advantage of the PSO algorithm is that the convergence speed is fast, the algorithm is simple, and it is easy to program.The disadvantage of the algorithm is that for the functions with multiple local extreme points, it is easy to fall into the local extremumpoints. Although the algorithm provides the possibility of global search, it cannot guarantee to converge to the global extremumpoints.

\subsection{Improved particle swarm optimization algorithm}

Based on the advantages of the original particle swarm optimization algorithm, this paper improves the convergence of the particle swarm optimization algorithm. It converges to the global best, and it avoids the problem that the global bestis replaced by the localextreme point.

When theinertia factor is 0 , combine the formula (5) and the formula (6) as (7) : 


$$
X_{\mathrm{id}}(t+1)=X_{i d}(t) c_{1} * \operatorname{rand}()\left(p_{i d}(t)-X_{i d}(t)\right)+c_{2}()\left(p_{g d}(t)-X_{i d}(t)\right) .
$$

According to the formula, only the position of the particles changes, and the flow is simplified.But it still maintains the linearly convergence.The main steps of the improved particle swarm algorithm are as follows:

Step1: Initialize the particle velocity and position, and set the inertia factor to 0 ;

Step2: Calculate the fitness value of a particle;

Step3: Calculate the historical optimal solution of the individual;

Step4: Calculate the global optimal solution;

Step5: Update the position and velocity of the particle according to the iterative formula;

Step6: Determine if the maximum number of iterations is reached, if not, go to (2);

Step7: End the algorithm and output the optimal solution.

\subsubsection{Initialization}

The initialization state of the PSO is a group of random particles (random solutions).The particles then seek the optimal solutions through iteration.During each iteration, particles update their velocity and position by tracking two "extreme values".

When the value of $W=0$, the first solution is the individual extremum (The first solution is the optimal solution found by the particle itself); the second solution is the global extremum (It is the optimal solution currently found for the entire population). The initialization process of the particle swarm algorithm is as follows:

(1) Set the population size $n$;

(2) For any values of $i$ and $j$ follow uniform distribution from $\left[-X_{\min }, X_{\max }\right]$;

(3) For any values of $i$ and $j$ follow uniform distribution from $\left[-V_{\min }, V_{\max }\right]$;

(4) For any $i$, let $y_{i}=x_{i}$.

where:

$j$ represents the $j$ th dimension of the particle;

$i$ represents the $i$ th particle;

$y_{i}$ and $x_{i}$ represent the optimal position and current position of the $i$ th particle.

\section{Experimental results and comparison}

\subsection{Sample set description and parameter Settings}

Set the initial value according to the problem in the literatures[2]. The central warehouse is represented by 0 , and the freight volume per vehicle is the value of $q=1.0$. There are a total of 3 cars to complete the mission.

The coordinate values of the warehouse and the freight volume for goods are shown in Table 1.

Table 1: Warehouse Coordinates and Shipping Volume

\begin{tabular}{|l|c|c|c|c|c|c|c|c|}
\hline $\begin{array}{l}\text { Serial } \\
\text { number }\end{array}$ & 0 & 1 & 2 & 3 & 4 & 5 & 6 & 7 \\
\hline Coordinate & $(18,54)$ & $(22,60)$ & $(58,69)$ & $(71,71)$ & $(83,46)$ & $(91,83)$ & $(24,42)$ & $(18,40)$ \\
\hline $\begin{array}{l}\text { Freight } \\
\text { Volume }\end{array}$ & & 0.89 & 0.14 & 0.28 & 0.33 & 0.21 & 0.41 & 0.57 \\
\hline
\end{tabular}

Initialize the particle swarm $n=40$, the inertia factor $w=0$, the learning factor $c_{1}=c_{2}=1.4944$, the maximum algebra is 100 , and the search space dimension (the number of unknowns) is 40 .The vehicle path model and the particle swarm algorithm are used to solve the problem.

Use the calculation formula to get the distance.

Suppose there are two points $A\left(x_{1}, y_{1}\right), B\left(x_{2}, y_{2}\right)$,then the distance between them is shown as (8).

$$
A B=\sqrt{\left(y_{2}-y_{1}\right)^{2}+\left(x_{2}-x_{1}\right)^{2}}
$$




\subsection{Experimental process}

When the inertia coefficient is 0 , the flight speed of the particle only depends on the current position of the particle, the besthistorical position of the particle, and the best historical position of the particle swarm.

Particles with the best global position will remain the current position, while the other particles will tend to move to the best position.So the particle swarm will shrink into the range of the best position in the space. According to the results of the code execution, when the number of iterations reaches 12 , the optimal value is obtained, and the optimal total distance of driving is 217.8135.

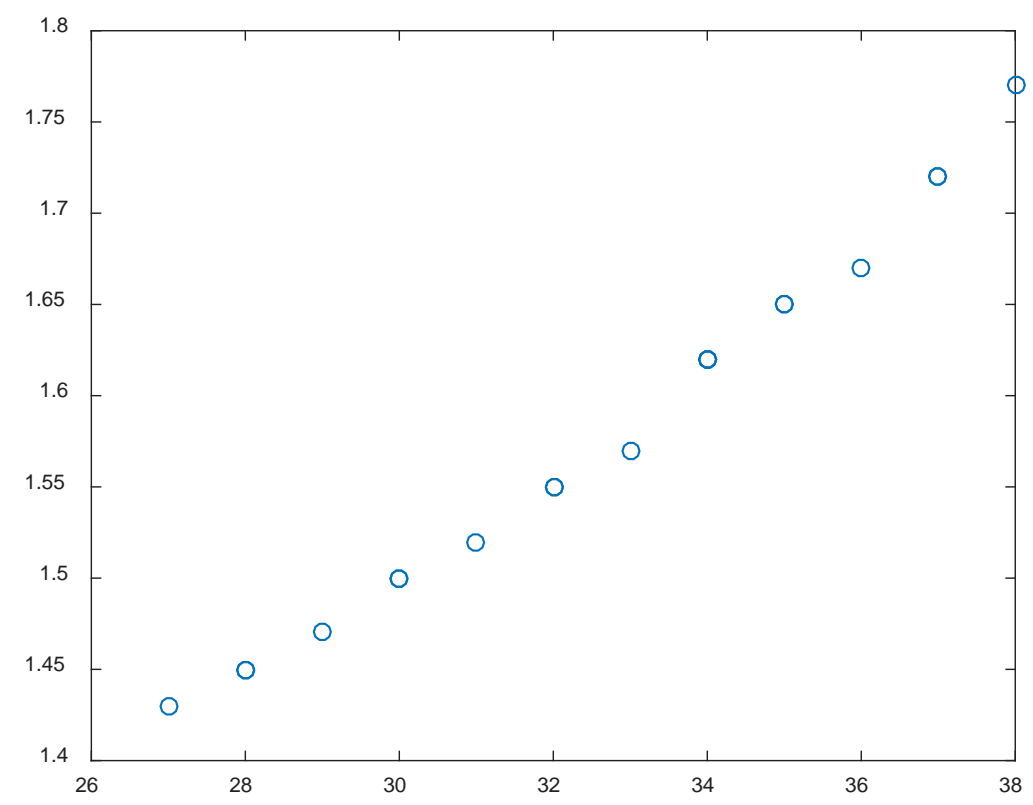

(a) Distribution of the final non-inferior solution in the target space before improvement

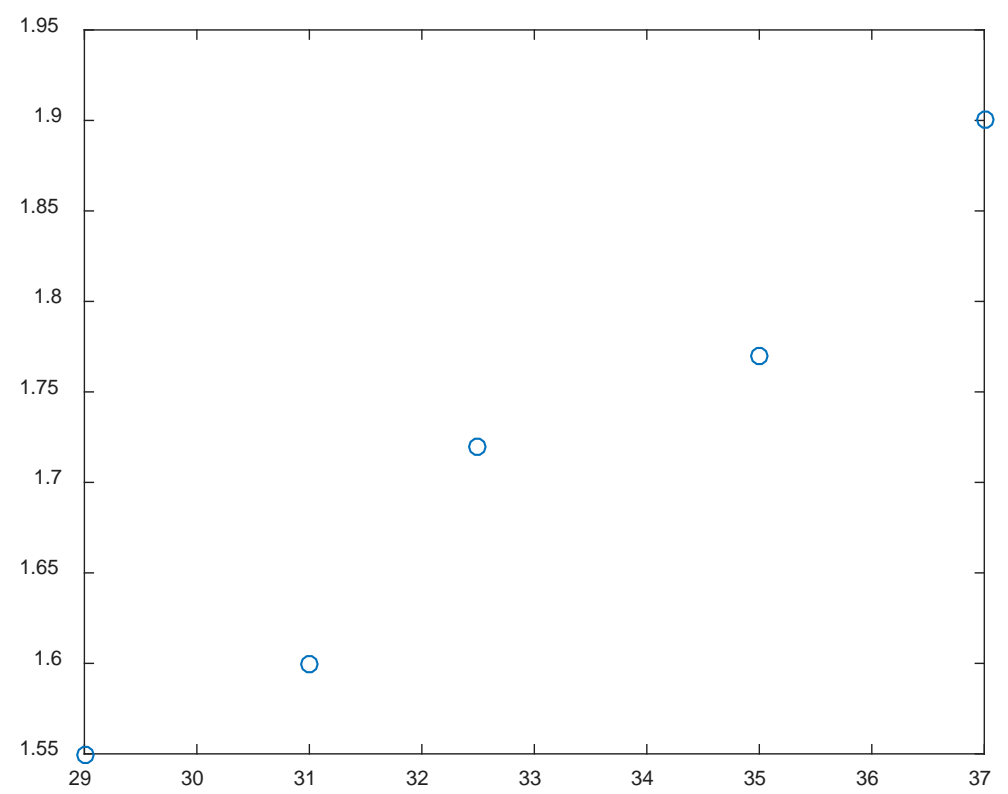

(b) The distribution of the final non-inferior solution in the target space after improvement

Figure 1: Distribution of the final non-inferior solution in the target space

According to Fig. 1, it can be concluded that the number of improved non-inferior solutions is reduced, and the entire evolution equation has a stronger local searching ability. The convergence of the improved particle swarm optimization algorithm increases.And the problemthat the optimal value is replaced by the local extremumvalue no longer occurs. 


\section{Summary}

Optimizing the logistics distribution vehicle path can not only improve the economic benefits of logistics, but also realize the scientific logistics and the optimization of human and material resources. This paper mainly improves the particle swarm optimization algorithm, the improved algorithm avoids the problems that the global optimal value is replaced by the local extremum and the optimal solution is difficult to find when searching.In addition, the convergence of the improved particle swarm optimization algorithm is increased, the number of iterations to obtain the optimal value is reduced, and the running time is shortened. The improved particle swarm optimization algorithm proposed in this paper is better than the unmodified particle swarm optimization algorithm. Therefore, the improved particle swarm optimization algorithm is an effective method to solve the optimal routing problem of logistics distribution vehicles.

\section{Acknowledgements}

Funding: This study was funded by Jilin Province Education Science"the13th Five-Year Plan " annual issue in 2017 (Grant Number GH170336).

\section{References}

[1] Dantzig, George Bernard, Ramser, John Huber. The Truck Dispatching Problem [J]. Management Science. 6 (1): 80-91.

[2] Yang Hulin. Research on improved particle swarm optimization algorithm for solving vehicle routing problem [D]. Guangxi Teachers College, 2012

[3] Qiu Ping. Research on Vehicle Routing Problem [D]. Dalian Maritime University, 2007.

[4] Sun Zhongyue. Research on Simulation Optimization Method of Vehicle Routing Problem [D]. Beijing Jiaotong University, 2012. 